Research Article

\title{
Study on Electroosmosis Consolidation of Punctiform Electrode Unit
}

\author{
Xiaoyu Yang $\mathbb{D}$, Yongbin Xie $\mathbb{D}$, Jianhua Dong $\mathbb{D}^{\mathbb{D}}$, Guosheng Liu, and Yalin Zheng \\ Western Centre of Disaster Mitigation in Civil Engineering, Ministry of Education, Lanzhou University of Technology, \\ Lanzhou 730050, China \\ Correspondence should be addressed to Jianhua Dong; djhua512@163.com
}

Received 5 January 2021; Revised 3 March 2021; Accepted 1 April 2021; Published 12 April 2021

Academic Editor: Hao Zheng

Copyright (C) 2021 Xiaoyu Yang et al. This is an open access article distributed under the Creative Commons Attribution License, which permits unrestricted use, distribution, and reproduction in any medium, provided the original work is properly cited.

In the electroosmosis method, when the distance between the opposite electrode and the same electrode is equal, the twodimensional effect of electroosmotic consolidation is significant, and the use of one-dimensional model will overestimate the potential gradient, making the calculated pore pressure value too large. Aiming at this problem, according to the electrode arrangement rule and the minimum composition, a punctiform electrode unit model is proposed, and electroosmotic experiments are carried out on the symmetric and asymmetric unit models. The two-dimensional electroosmotic consolidation governing equation of the punctiform electrode unit is established. The electric potential field of the electrode unit and the finite element form of the electroosmotic consolidation equation are given by the Galerkin method. The PyEcFem finite-element numerical library is developed using Python programming to calculate. The research results show the following: (1) The two-dimensional effect of the potential field distribution of the punctiform electrode unit is significant. The reduction of spacing of the same nature electrode in the symmetrical unit can make the potential distribution close to a uniform electric field. The asymmetry prevents the electric potential field distribution from being reduced to a one-dimensional model. (2) The number of anodes will affect the electroosmosis effect of the soil. The more the anodes, the better the electroosmosis reinforcement effect of the soil, and the distribution of negative excess pore water pressure will be more uniform. (3) In the early stage of electroosmosis, the more the drainage boundaries, the faster the generation of negative pore pressure, but in the middle and late stages of electroosmosis, the potential value becomes the decisive factor, and the amplitude of negative pore water pressure in asymmetric units is higher than that in symmetric units. The potential distribution will not affect the degree of consolidation but will affect the extreme pore water pressure.

\section{Introduction}

Electroosmosis drainage reinforcement method is a good method to deal with low-permeability soft soil [1]. In 1939, Casagrande [2] applied this method to practical engineering for the first time and achieved success. Since then, it had attracted the attention of many scholars and carried out a large number of experimental studies. These experimental studies mainly focused on the influencing factors of electroosmosis method [3], the characteristics of soil after electroosmosis treatment [4], and how to improve the efficiency of electroosmosis method [5]. On the basis of previous experimental research experience, the subsequent scholars further carried out the theoretical research of electroosmotic consolidation. The theory of electroosmotic consolidation greatly promoted the application of electroosmotic method and deepened people's understanding of electroosmotic method.

One-dimensional electroosmotic consolidation theory was proposed by Esrig [6], which is based on Terzaghi's seepage consolidation principle. Electroosmotic and hydraulic seepage meet the linear superposition principle, and the dissipation law of excess pore water pressure under electroosmotic action is given. Most of the subsequent researches on electroosmotic consolidation theory are based on the classical Esrig theory. Lewis and Humpheson [7] introduced the change of the current and solved the electroosmotic consolidation theory. Wan and Mitchell [8] 
established the theory of electroosmotic consolidation under surcharge condition and demonstrated the feasibility of electrode reversal, which can effectively reduce the energy consumption of electroosmotic. Shang [9] proposed a twodimensional theoretical model of vertical electroosmosis under surcharge condition, which can effectively improve the soil reinforcement effect through the combination of surcharge and electroosmosis. Su and Wang [10] extended the one-dimensional electroosmotic consolidation theory to the two-dimensional model based on the idea of partition and initially established the two-dimensional electroosmotic consolidation theory. Li et al. [11] established the electroosmotic consolidation equation in the axisymmetric model and gave the analytical solution, which further developed the consolidation theory of surcharge combined electroosmotic method. Wu et al. [12] based on Biot's theory, the stress of soil under electroosmosis was calculated, and the change of soil properties caused by chemical change was considered in the model. During the electroosmosis drainage process, the soil gradually changed from the initial saturated state to the unsaturated state, so the saturation changed. The electroosmotic consolidation model considering the change of saturation is proposed [13], which makes the calculation results closer to the real situation. On the basis of the above saturation changes, the large deformation theory is introduced to improve the influence of soil properties changes in the process of electroosmotic consolidation by Deng and Zhou [14] and Zhou et al. [15-17]; the change of soil saturation also attracted the attention of Wang et al. [18]. In the twodimensional model, the vacuum preloading method was introduced into the electroosmotic consolidation model, and the governing equation of this case was given and solved analytically by Wang et al. [19]. Sheng et al. [20] carried out experimental research on the two-dimensional potential distribution of electroosmotic under the action of plastic electrode plate, and the two-dimensional electroosmotic consolidation was calculated through the measured values. The potential attenuation in the process of electroosmosis will lead to the increase of energy consumption, which is discussed by Yang and Dong [21]. These studies improve the theory of electroosmotic consolidation and provide theoretical support for the engineering application of electroosmotic method. However, most of the existing researches on electroosmotic consolidation theory are based on one-dimensional model. Electroosmotic consolidation is largely affected by the electrode arrangement. When the same electrode spacing is equal to that of the opposite electrode, the potential attenuation of soil between the same electrode is more obvious, and the twodimensional effect is significant. If one-dimensional model is used to describe the electroosmotic consolidation, the deviation caused by this simplification is not obvious at present in essence. The two-dimensional electroosmotic consolidation model based on the idea of partition can only be applied to the electroosmosis with parallel arrangement and cannot be used to calculate the electrode polarity in the case of asymmetry. These problems are still in lack of detailed and in-depth quantitative research. Therefore, it is urgent to establish a two-dimensional electroosmotic consolidation theory which can describe the electrode arrangement.

In this paper, on the basis of previous studies, according to the electrode composition and electrode polarity, the parallel and rectangular electrode arrangement is transformed into a punctiform electrode unit model. Taking the punctiform electrode unit as the research object, the electroosmotic consolidation tests of symmetric unit and asymmetric unit are carried out, and the potential distribution and pore pressure are measured. According to the punctiform electrode unit model, the two-dimensional electroosmotic consolidation equation is established, and the corresponding initial conditions and boundary conditions are given. The governing equation is discretized by Galerkin's method, which expresses the finite element form of governing equation. Python is used to program the numerical calculation library for solving the electroosmotic consolidation of punctiform electrode unit. The two-dimensional potential field and pore pressure field of electroosmosis are calculated. These researches on electroosmotic consolidation of punctiform electrode unit can provide some theoretical support for the engineering application of electroosmotic method.

\section{Materials and Methods}

2.1. Experimental Materials. In order to study the potential distribution and pore water pressure distribution of electrode unit with different electrode composition, two groups of experiments were carried out. The first model is symmetrical unit, which has two anodes and two cathodes. The second group contains three anodes and one cathode. The length and the width of the model box are all $50 \mathrm{~cm}$, and the height is $25 \mathrm{~cm}$, which is made of plexiglass. There are four electrode rod sockets at the corresponding position at the bottom of the model box, and a $40 \mathrm{~cm} \times 40 \mathrm{~cm}$ rectangle is formed between the electrodes (see Figure 1). The metal probe is made of iron wire with a height of about $22 \mathrm{~cm}$, which is used to measure the potential value at a certain point of the soil. Four probes about $1 \mathrm{~cm}$ away from the electrodes are used to measure the interface loss voltage in the process of electroosmosis. The rest of the metal probes are rectangular distribution, with vertical and horizontal spacing of $13.33 \mathrm{~cm}$ (see Figure 2(a)). The anode is made of iron metal rod with a diameter of $20 \mathrm{~mm}$, the cathode is made of stainless-steel tube with an outer diameter of $20 \mathrm{~mm}$, and the electrode length is $22 \mathrm{~cm}$ (see Figure 2(b)). There are drainage holes on the wall of the cathode tube for the discharge of pore water. The water-collecting box is used to collect the water from the cathode, and the quality of the discharged water can be measured in real time through an electronic scale. Micropore water pressure sensors are used to measure pore pressure during electroosmosis, and the maximum range of sensor is from $-100 \mathrm{kPa}$ to $100 \mathrm{kPa}$.

The other devices used include a DC power supply (PS$605 \mathrm{D})$ and a multimeter. The maximum output voltage of the DC power supply was $60 \mathrm{~V}$, and the maximum output 


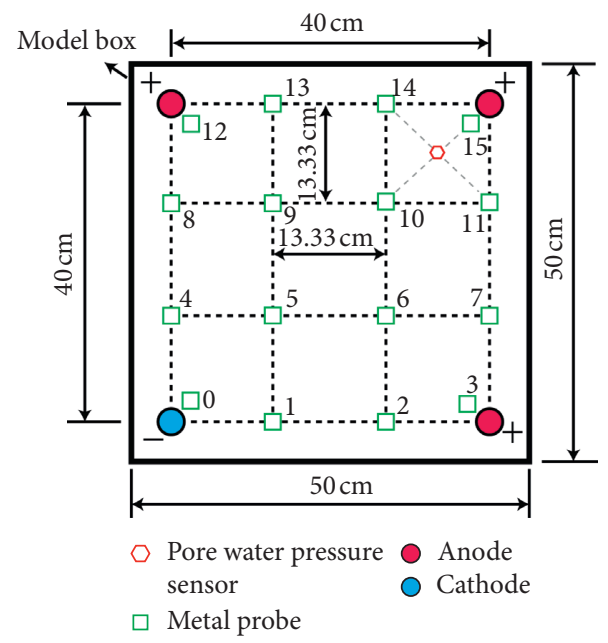

FIGURE 1: Schematic diagram of electroosmosis test device.

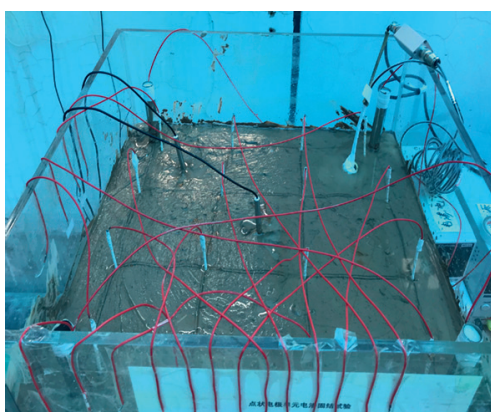

(a)

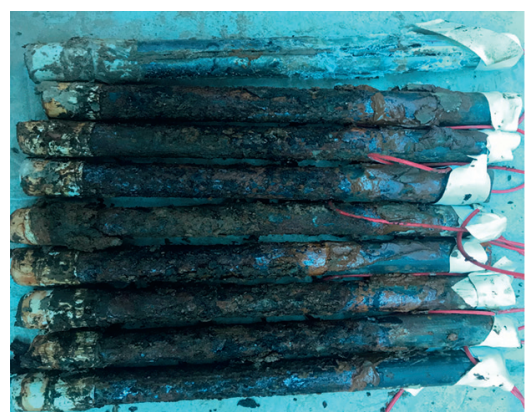

(b)

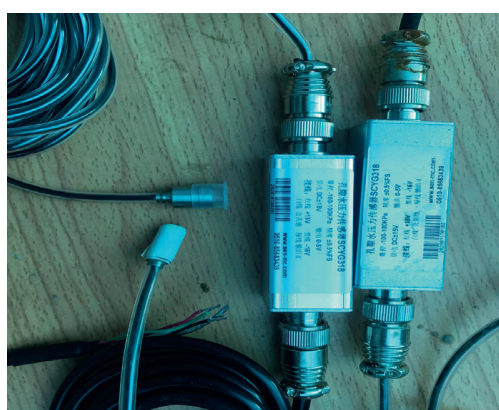

(c)

Figure 2: Electroosmotic consolidation model test. (a) Electroosmosis model box. (b) Metal electrodes. (c) Micropore water pressure sensors.

current was $3 \mathrm{~A}$. The multimeter was used to measure the potential value of the soil.

The soil sample used in the test is remolded silt soil, which is taken from a foundation pit in Lanzhou. The particle size distribution of the soil is shown in Figure 3. The initial moisture content of the soil sample is $29.37 \%$, the filling height of the soil sample in the test is $20 \mathrm{~cm}$, and the soil parameters are shown in Table 1.

2.2. Experimental Methods. The experimental study contains two experimental models, one is the electroosmosis test of the symmetric unit, and the other is the electroosmosis test of the asymmetric unit. In the asymmetric test group, due to the development of soil cracks, the pore pressure sensor became 0 at the 51st hour. Therefore, the electroosmosis duration of the two groups of tests was determined to be 51 hours. Both sets of test power supply voltages are $48 \mathrm{~V}$. Except for the electrode polarity, the other test parameters are the same in the two experimental models.

First of all, the soil sample should be treated. The silt soil was dried and crushed to remodel, and water was added to make the soil saturated. After resting for 48 hours, the average moisture content of the soil sample was about

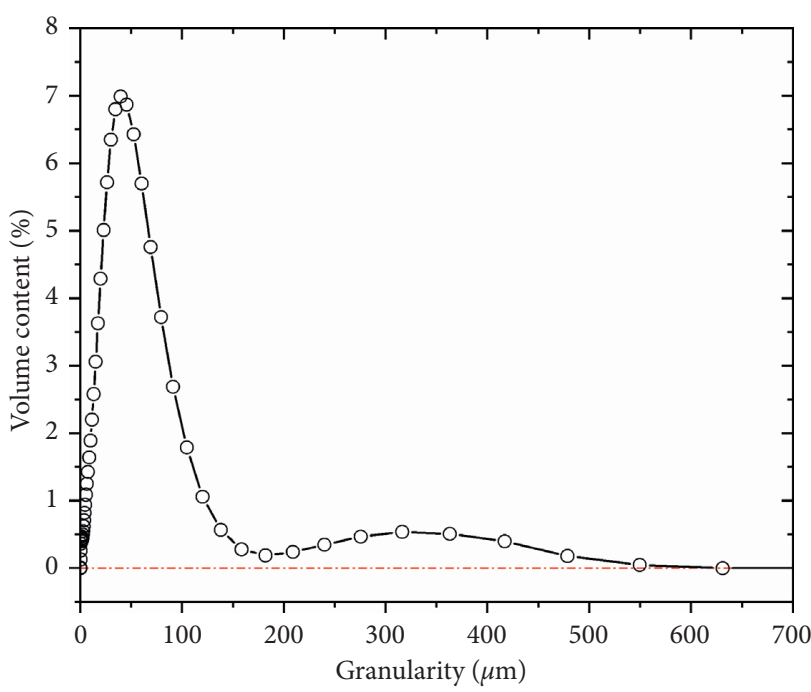

FIGURE 3: Soil particle size distribution curve.

TABLE 1: Physical properties of soil samples.

Specific gravity Liquid limit (\%) $\quad$ Plastic limit (\%) $\quad$ Saturation

\begin{tabular}{llll}
\hline 2.57 & 27 & 17 & 0.96 \\
\hline
\end{tabular}


$29.37 \%$, and soil sample saturation was 0.96 . The electrode was inserted into the small hole in the model box for fixing, and the electrodes were connected with the DC power supply through the wire. The soil was layered into the model box, and the sample height was $20 \mathrm{~cm}$. The water discharged in the process of electroosmosis flowed out from the cathode and was collected by the water collecting box. The reading interval of drainage quality was two hours, and the measurement is carried out in the daytime, meanwhile. The potential of each metal probe was measured by a multimeter to describe the potential distribution. The pore pressure sensor could continuously record the pore pressure in real time, and the sampling frequency was $1 / 60 \mathrm{~Hz}$. After the electroosmosis test, the moisture content of soil was measured at each metal probe position.

\section{Experiment Results}

The amount of pore water discharged during the electroosmosis process can be read through an electronic scale. The soil samples used in the two experiments and the applied voltage are the same. There is only one cathode in the symmetrical unit, and the pore water is discharged at the cathode. In the asymmetric unit, there are two cathodes, and pore water converges at the two cathodes. Figure 4(a) shows the change curve of the quality of discharged water during the electroosmosis process. The drainage rate in the symmetric unit was slightly higher than that in the asymmetric unit before the first 30 hours, but the deviation was smaller. After 30 hours, the drainage rate of the symmetrical unit decreased quickly. After the test, the quality of the discharged water from the asymmetrical unit was slightly higher than that of the symmetrical unit by about $6.9 \%$. During the electroosmosis process, the current generally showed a decreasing trend. However, in the first 8 hours, the currents of the two groups of tests showed an increase (see Figure 4(b)). This phenomenon was caused by the salt content of the soil sample, which was also discovered by other researchers [22]. The current began to decrease after reaching the peak value; at the end of the electroosmosis, the current was lower than the current value at the beginning of the test. The current of the symmetric unit was reduced by $31.8 \%$, and the current of the asymmetric unit was reduced by $44.5 \%$. The drainage effect of asymmetric units is higher than that of symmetric units. The more the anodes, the better the effect of electroosmosis reinforcement. The water content at different positions of the soil after electroosmosis also confirms this conclusion, as shown in Table 2.

A metal probe is placed about $1 \mathrm{~cm}$ away from the electrode to measure the interface voltage in the electroosmotic model. Figure 5 is a graph of potential changes at positions $0,3,12$, and 15 of the metal probes. In the symmetrical unit test, the electric potentials of the two cathodes are relatively consistent, and both attenuate with the progress of electroosmosis, with a decrease of $76.9 \%$. At the probes near the two anodes, the electric potentials also show a decreasing trend, with an average decrease amplitude is $34.3 \%$ (see Figure 5(a)). In the asymmetric unit, the distance between the three anodes and the cathode is different, which makes the pore water seepage path different. The anode with a longer distance will have a lower drainage rate than the other two anodes, and the moisture content of the soil will affect the soil. Therefore, the potential at the anode farther away increases with the progress of electroosmosis, and the amplitude is about $16.4 \%$. The other two anode potentials are decreasing, with an average decrease of $40.8 \%$ (see Figure 5(b)). There is only one cathode in the asymmetric unit, and its potential value is reduced by $61.4 \%$.

The effective voltage is the voltage that is really applied to the soil. The contact voltage between the electrode and the soil will make the actual electroosmotic voltage to be lower than the power supply voltage. This problem will increase the energy consumption of electroosmosis. The potential values at several metal probes are converted into an effective voltage; that is, the zero-potential-energy surface is selected as the probe at the cathode to obtain the effective voltage change of the electroosmosis test, as shown in Figure 6. The effective voltage in the symmetrical unit continues to decrease, with an average decrease of $25.4 \%$ (see Figure $6(a)$ ). The effective voltage decreases faster in the early stage of electroosmosis and changes more slowly in the later stage. In the asymmetric unit, the effective voltage of anode 1 and anode 2 (see Figure 6(b)) showed a downward trend, with an average decrease of $35.8 \%$, while the effective voltage of anode 3 , which is farther from the cathode, increased by $44.9 \%$.

The metal probes arranged in the box can obtain the potential distribution of the electrode unit. Figure 7 shows the measured potential distribution of the unit at the beginning of electroosmosis. In the symmetric unit (see Figure $7(\mathrm{a})$ ), the electrodes are arranged symmetrically, so the potential distribution has good symmetry, and the potential gradient changes little near the cathode. In the asymmetric unit (see Figure $7(b)$ ), the reduction of the potential is affected by the electrode arrangement and does not have a symmetrical characteristic. When the distance between the same electrode and the opposite electrode of the punctiform electrode unit is equal, the electric potential distribution of the unit has a strong two-dimensional characteristic, and the electric field distribution is significantly different from a uniform electric field.

Figure 8 shows the measured pore water pressure during the electroosmosis process. The change rate of the pore pressure of the symmetric unit is lower than that of the asymmetric unit. The asymmetric unit contains three anodes, and the drainage rate is faster. The more the anodes, the better the soil electroosmosis effect. The pore pressure of the symmetric unit changes from $1.08 \mathrm{kPa}$ to $-17.01 \mathrm{kPa}$, and the pore pressure of the asymmetric unit changes from $1.465 \mathrm{kPa}$ to $-21.53 \mathrm{kPa}$. With the same electroosmosis conditions and time, the reinforcement effect of asymmetric units is better than that of symmetric units. The more the anodes, the better the electroosmosis drainage effect.

\section{Electroosmotic Consolidation Equation and Calculation Method}

4.1. Governing Equation. The electroosmosis test of the punctiform electrode unit shows that there is a clear 


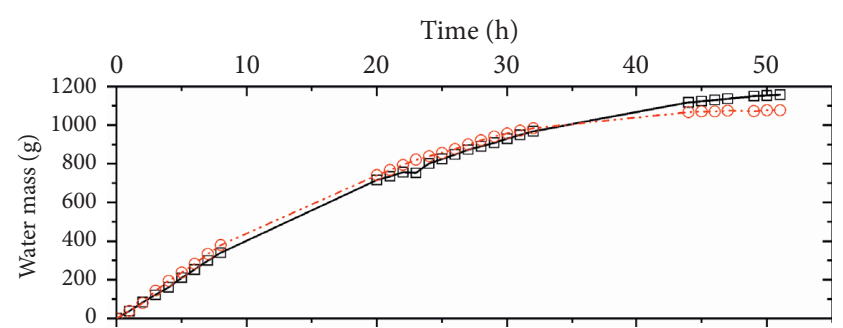

(a)

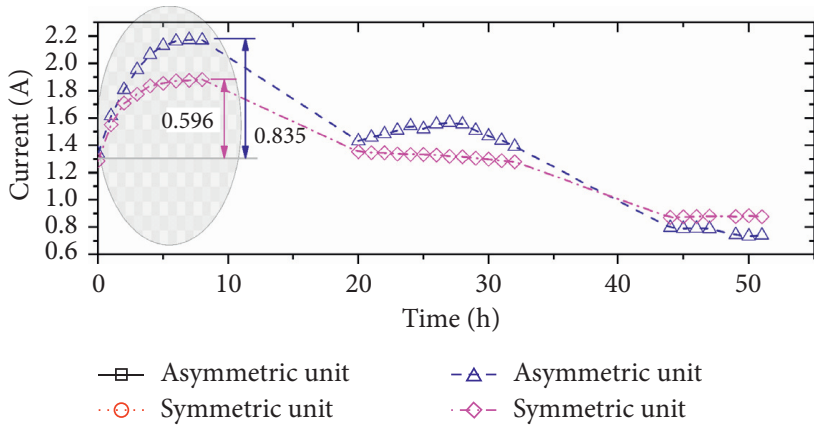

(b)

Figure 4: Test of the actual displacement and current.

TABLE 2: Soil moisture content on the diagonal of the unit.

\begin{tabular}{lcccc}
\hline Test model & Position $15(\%)$ & Position $10(\%)$ & Position 5 (\%) & Position 0 (\%) \\
\hline Symmetric unit & 19.64 & 20.65 & 22.27 & 26.72 \\
Asymmetric unit & 18.42 & 19.68 & 21.14 & 26.39 \\
\hline
\end{tabular}

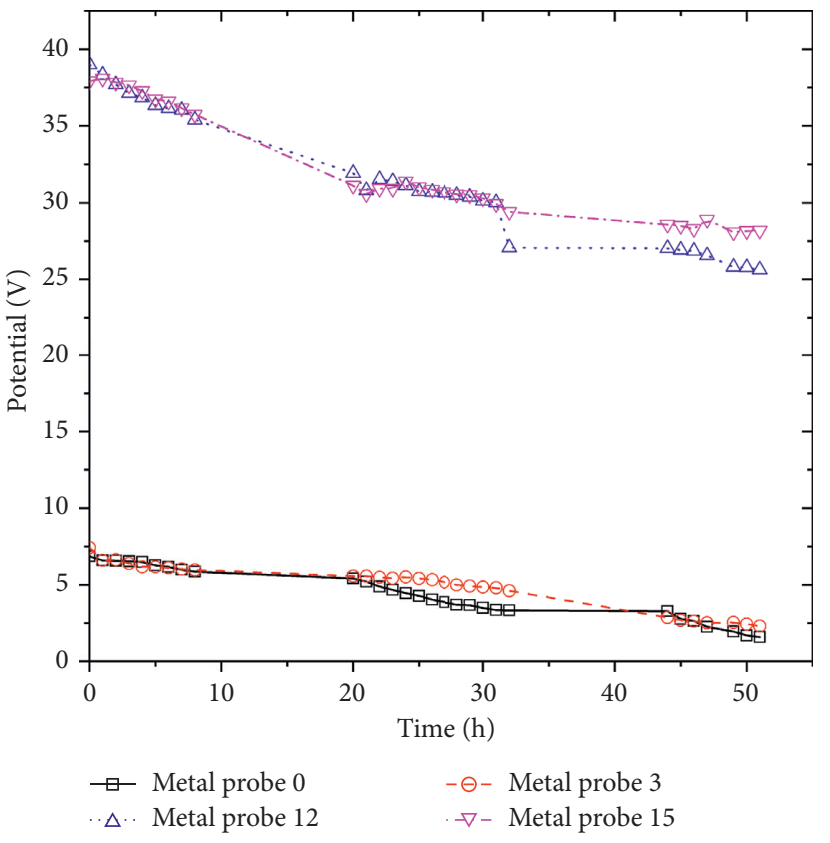

(a)

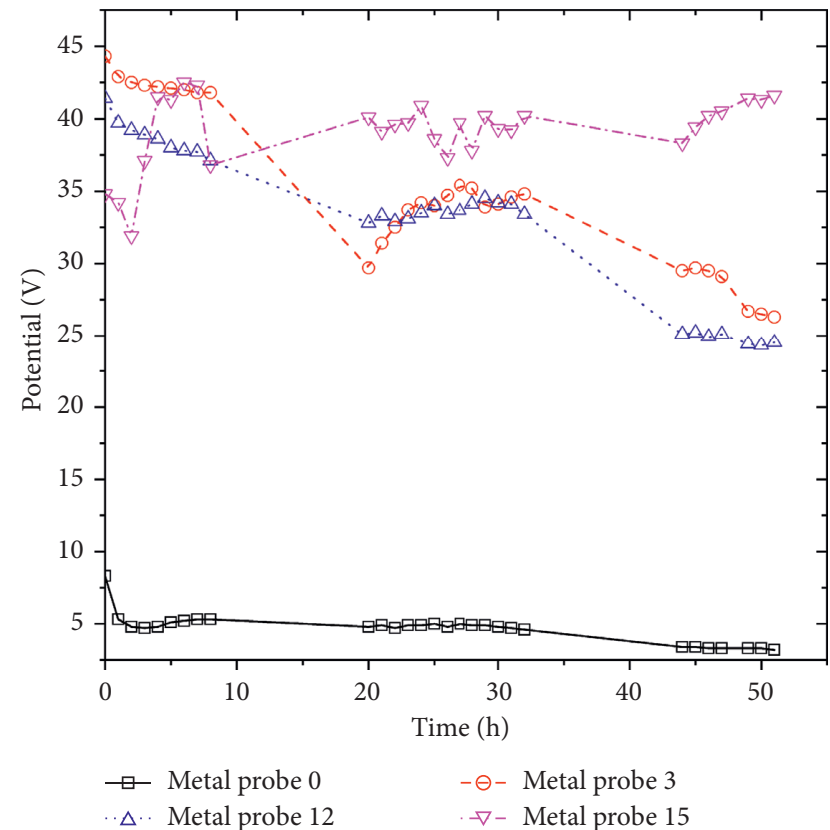

(b)

FIgURE 5: Electrode potential change curve. (a) Symmetrical unit model. (b) Asymmetrical element model.

difference between the electric potential distribution and the uniform electric field. If the one-dimensional electroosmotic consolidation theory is used for calculation, the influence of the electric potential distribution on the pore pressure cannot be reflected. Therefore, it does have positive significance to establish the two-dimensional unit theory of electroosmotic consolidation. The model still satisfies the assumptions in the Esrig theory, the soil is considered to be isotropic, the hydraulic seepage meets Darcy's law, and the relationship between the electroosmosis and hydraulic seepage meets the linear superposition principle. 


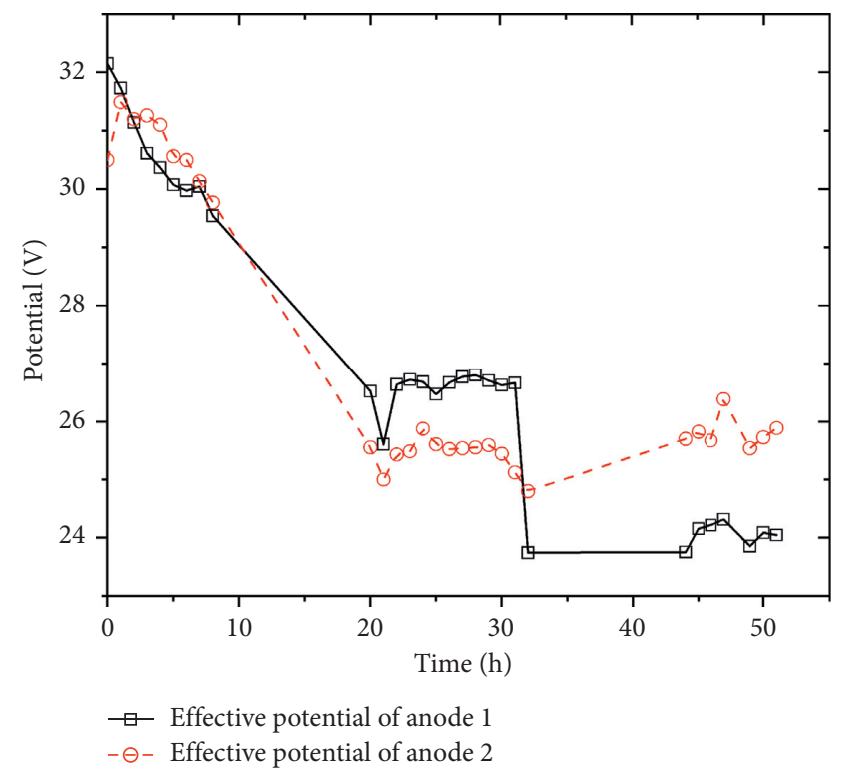

(a)

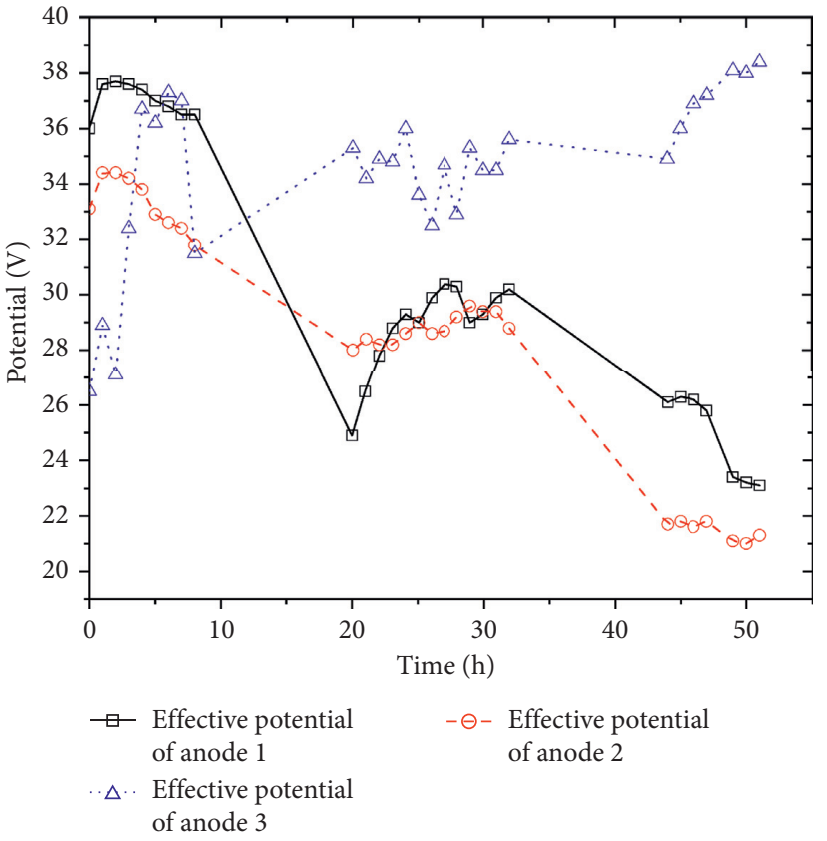

(b)

Figure 6: Effective potential change curve. (a) The effective potential of the symmetric unit. (b) The effective potential of the asymmetric unit.

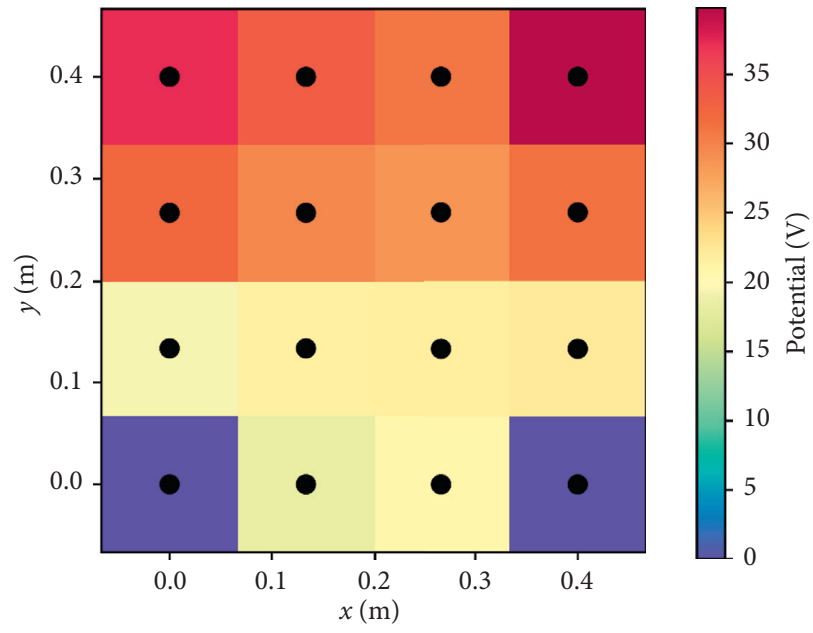

(a)

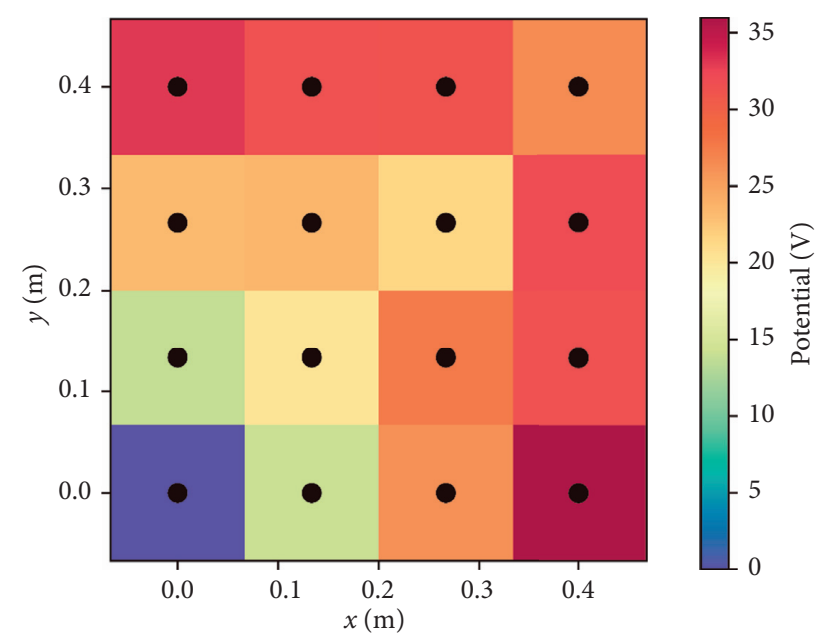

(b)

Figure 7: Potential distribution of test model. (a) The potential distribution of the symmetric unit. (b) The potential distribution of the asymmetric unit.

The change in soil volume is equal to the volume of pore water flowing out. The outflow of pore water of the microelement body per unit time is shown in the following equation:

$$
\Delta \mathrm{Q}=\frac{\partial v_{x}}{\partial x} \mathrm{~d} x \cdot(\mathrm{d} y \cdot 1)+\frac{\partial v_{y}}{\partial y} \mathrm{~d} y \cdot(\mathrm{d} x \cdot 1),
$$

where $\Delta Q$ is the volume of water flowing out per unit time, $v_{x}$ is the seepage velocity in the $x$-direction, $v_{y}$ is the seepage velocity in the $y$-direction, and $\mathrm{d} x$ and $\mathrm{d} y$ are the lengths of the infinitesimal body in the $x$ - and $y$-directions, respectively.

The change of soil volume per unit time $\Delta V$ is as follows:

$$
\Delta V=\frac{\partial \varepsilon_{v}}{\partial \mathrm{t}}(\mathrm{d} x \cdot \mathrm{dy} \cdot 1)=-m_{v} \frac{\partial \mathrm{u}}{\partial \mathrm{t}}(\mathrm{d} x \cdot \mathrm{d} y \cdot 1),
$$

where $\Delta V$ is the change in soil volume per unit time. $\varepsilon_{v}$ is the volumetric strain of the soil. $m_{v}$ is the volumetric 


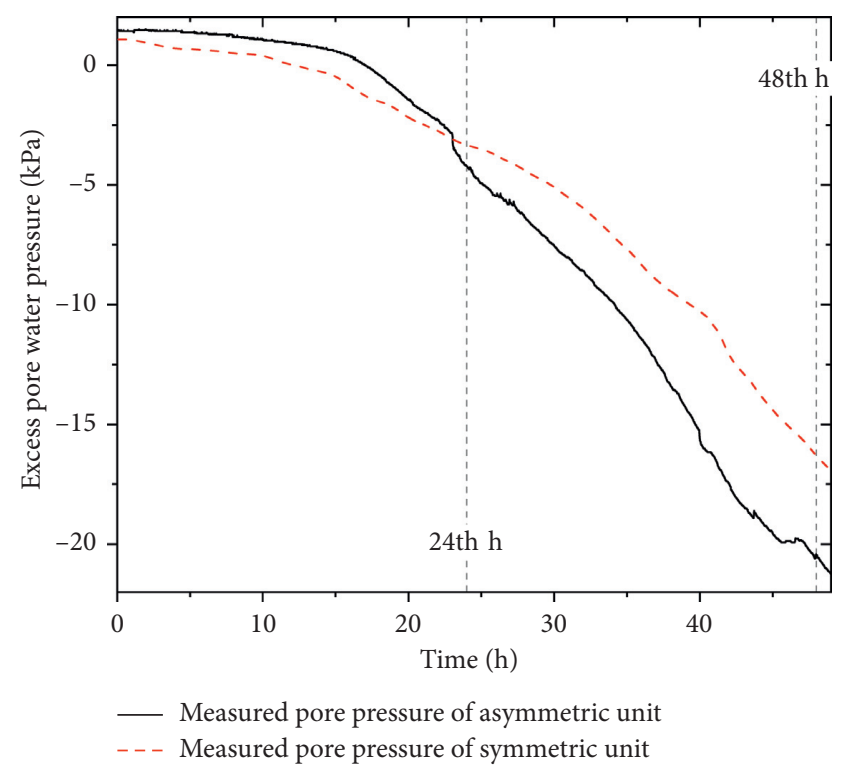

FIgURE 8: Picture of electroosmotic excess pore water pressure.

compressibility of the soil. $u(x, y, t)$ is the pore water pressure function.

According to the principle of water flow continuity, $\Delta V=\Delta Q$, the following equation can be obtained from formulas (1) and (2):

$$
\frac{\partial v_{x}}{\partial x}+\frac{\partial v_{y}}{\partial y}=-m_{v} \frac{\partial u}{\partial t}
$$

In the process of electroosmosis, both hydraulic seepage and electroosmosis will cause the flow of pore water. These two flows satisfy the principle of linear superposition. The soil permeability coefficient is equal in two directions, and the electric permeability coefficient is the same in both directions. Equation (4) is as follows:

$$
v_{x}=v_{h x}+v_{e x}=-\left(\frac{k_{h}}{\gamma_{w}} \frac{\partial u}{\partial x}+\frac{\partial \phi}{\partial x}\right),
$$

where $v_{x}$ is the velocity of hydraulic seepage in the $x$-direction, $v_{e x}$ is the water flow velocity caused by the electric potential gradient in the $x$-direction, $k_{h}$ is the permeability coefficient of soil, $k_{e}$ is the electric permeability coefficient, and $\gamma_{w}$ is the weight of water, and $\Phi$ is the potential function.

In the same way, the speed expression in the $y$-direction can be obtained in the following form:

$$
v_{y}=v_{h y}+v_{e y}=-\left(\frac{k_{h}}{\gamma_{w}} \frac{\partial u}{\partial y}+\frac{\partial \phi}{\partial y}\right) .
$$

Substituting formula (4) and (5) into (3), the twodimensional electroosmotic consolidation governing equation can be obtained:

$$
\frac{\partial^{2} u}{\partial x^{2}}+\frac{\gamma_{w} k_{e}}{k_{h}} \frac{\partial^{2} \phi}{\partial x}+\frac{\partial^{2} u}{\partial y^{2}}+\frac{\gamma_{w} k_{e}}{k_{h}} \frac{\partial^{2} \phi}{\partial y^{2}}=\frac{m_{v} \gamma_{w}}{k_{h}} \frac{\partial u}{\partial t}
$$

In order to solve the governing equation, the imaginary $\zeta(x, y, t)$ function is introduced to transform equation (6). The $\zeta(x, y, t)$ expression is shown in the following equation:

$$
\zeta(x, y, t)=u(x, y, t)+\frac{\gamma_{w} k_{e}}{k_{h}} \phi(x, y) .
$$

Combining equation (7) with equation (6), the governing equation of $\zeta(x, y, t)$ can be expressed:

$$
C_{h}\left(\frac{\partial^{2} \zeta}{\partial x^{2}}+\frac{\partial^{2} \zeta}{\partial y^{2}}\right)=\frac{\partial \zeta}{\partial t}
$$

where $C_{h}$ is the soil consolidation coefficient, $C_{h}=k_{h} / m_{v} \gamma_{w}$.

Equation (8) is the governing equation for two-dimensional electroosmosis consolidation. This equation is a parabolic partial differential equation. The equation can be solved by combining boundary conditions and initial conditions. After solving $\zeta(x, y, t)$, equation (6) can be used to calculate the pore water pressure distribution $u(x, y, t)$.

4.2. Boundary Conditions and Initial Conditions. The twodimensional electroosmotic consolidation model of the electrode unit is shown in Figure 9. The origin of the coordinate system is point $O$, the length in the $x$-direction is $L$, and the width in the $y$-direction is $W$.

In the punctiform electrode unit, the geometric size of the electrode is much smaller than the size of the unit, so the electrode can be regarded as a point. The opening of the cathode can drain water. At the cathode, the pore water pressure is 0 and the potential value is 0 , which meets the Dirichlet condition. The anode is an undrained boundary, and the seepage velocity at the anode is 0 , which satisfies the Neumann boundary condition. The points on the boundary other than the electrode position are unconstrained. The boundary conditions of the symmetric unit can be obtained as follows:

$$
\begin{aligned}
\zeta(0,0, t)= & u(0,0, t)+\frac{\gamma_{w} k_{e}}{k_{h}} \Phi(0,0)=0, \\
\zeta(L, 0, t)= & u(L, 0, t)+\frac{\gamma_{w} k_{e}}{k_{h}} \Phi(L, 0)=0, \\
& \zeta_{x}(0, W, t)+\zeta_{y}(0, W, t)=0, \\
& \zeta_{x}(L, W, t)+\zeta_{y}(L, W, t)=0 .
\end{aligned}
$$

The boundary conditions of the asymmetric unit are

$$
\begin{array}{r}
\zeta(0,0, t)=u(0,0, t)+\frac{\gamma_{w} k_{e}}{k_{h}} \Phi(0,0)=0, \\
\zeta_{x}(L, 0, t)+\zeta_{y}(L, 0, t)=0, \\
\zeta_{x}(L, W, t)+\zeta_{y}(L, W, t)=0, \\
\zeta_{x}(0, W, t)+\zeta_{y}(0, W, t)=0 .
\end{array}
$$




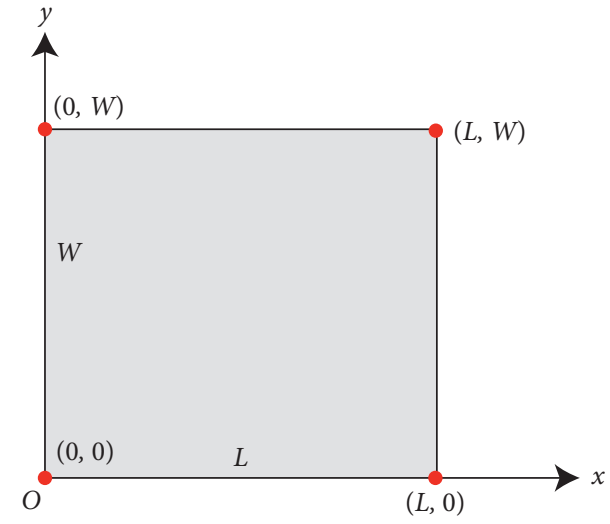

Figure 9: The electrode unit model.

According to formula (7), the initial conditions of the $\zeta(x, y, t)$ equation can be obtained. The initial conditions of both the symmetric unit and the asymmetric unit can be expressed as follows:

$$
\zeta(x, y, 0)=u(x, y, 0)+\frac{\gamma_{w} k_{e}}{k_{h}} \Phi(x, y)
$$

4.3. The Finite-Element Form. The initial conditions include the electric potential distribution function $\Phi(x, y)$. To solve the two-dimensional electroosmotic consolidation problem, the electric potential field must be calculated first. In onedimensional electroosmotic consolidation, the electric field is a uniform electric field, and the potential distribution is linearly reduced. But for the punctiform electrode unit, it is necessary to solve the boundary value problem under the action of the point electrode based on the electrostatic field theory. Since the boundary conditions of the potential field and the pore pressure field in the two-dimensional electroosmosis model of the punctiform electrode unit are in the form of discrete points, it is difficult to use analytical solutions. The finite element method is used here for obtaining numerical calculation. For the electric potential field, it satisfies the Laplace equation; see the following equation:

$$
\frac{\partial^{2} \Phi}{\partial x^{2}}+\frac{\partial^{2} \Phi}{\partial y^{2}}=0
$$

The Galerkin method is used to discretize the equation. The finite-element shape is a three-node triangular element, and the corresponding shape function is

$$
\Phi(x, y)=\left\{\begin{array}{lll}
N_{1} & N_{2} & N_{3}
\end{array}\right\} \cdot\left\{\begin{array}{lll}
\varphi_{1} & \varphi_{2} & \varphi_{3}
\end{array}\right\}^{T}=\{\mathbf{N}\} \cdot\{\boldsymbol{\varphi}\}^{T},
$$

where $\{\mathbf{N}\}$ is the shape function vector and $\{\varphi\}$ is the potential vector at the node of the element.

The strong formulation of equation (12) is

$$
\iint_{\Omega}\{\mathbf{N}\}^{T} \cdot\left(\frac{\partial^{2} \Phi}{\partial x^{2}}+\frac{\partial^{2} \Phi}{\partial y^{2}}\right) \mathrm{d} x \mathrm{~d} y=0
$$

Applying Green's formula to equation (12), when it is a homogeneous boundary condition, the weak form corresponding of Laplace equation can be obtained:

$$
\iint_{\Omega}\left(\frac{\partial\{\mathbf{N}\}^{T}}{\partial x} \frac{\partial \Phi}{\partial x}+\frac{\partial\{\mathbf{N}\}^{T}}{\partial y} \frac{\partial \Phi}{\partial y}\right) \mathrm{d} x \mathrm{~d} y=0
$$

Combining equations (13) and (15), the finite-element form of the electric potential field governing equation is obtained:

$$
\iint_{\Omega}\left(\frac{\partial\{\mathbf{N}\}^{T}}{\partial x} \frac{\partial\{\mathbf{N}\}}{\partial x}+\frac{\partial\{\mathbf{N}\}^{T}}{\partial y} \frac{\partial\{\mathbf{N}\}}{\partial y}\right) \mathrm{d} x \mathrm{~d} y \cdot\{\boldsymbol{\varphi}\}^{T}=0 .
$$

The stiffness matrix of the element can be obtained by formula (16), and the total stiffness matrix is obtained through integration. The electric potential boundary condition is the Dirichlet condition, which is introduced into the linear equation system through the Lagrangian penalty function method, so that the electric potential field of the element can be calculated distributed. On the basis of the electric potential field distribution, the initial conditions of the electroosmotic consolidation equation (8) can be determined. The governing equation of the pore water pressure field is an unsteady state equation. First, use the Galerkin method to discretize the spatial items of the equation to obtain the corresponding time $i$ expression:

$$
\begin{gathered}
\iint\left(\frac{\partial\{\mathbf{N}\}^{T}}{\partial x} \frac{\partial\{\mathbf{N}\}}{\partial x}+\frac{\partial\{\mathbf{N}\}^{T}}{\partial y} \frac{\partial\{\mathbf{N}\}}{\partial y}\right) \mathrm{d} x \mathrm{~d} y \cdot\left\{\mathbf{u}_{i}\right\}^{T} \\
+\iint\{\mathbf{N}\}^{T} \cdot\{\mathbf{N}\} \mathrm{d} x \mathrm{~d} y \cdot \frac{\mathrm{d}\left\{\mathbf{u}_{i}\right\}^{T}}{\mathrm{~d} t}=0 .
\end{gathered}
$$

Let $\quad[\mathbf{k}]=\iint\left(\left(\partial\{\mathbf{N}\}^{T} / \partial x\right)(\partial\{\mathbf{N}\} / \partial x)+\left(\partial\{\mathbf{N}\}^{T} / \partial y\right)\right.$ $(\partial\{\mathbf{N}\} / \partial y)) \mathrm{d} x \mathrm{~d} y,[\mathbf{s}]=\iint\{\mathbf{N}\}^{T} \cdot\{\mathbf{N}\} \mathrm{d} x \mathrm{~d} y$, equation (17) can be expressed as follows:

$$
[\mathbf{k}] \cdot\left\{\mathbf{u}_{i}\right\}^{T}+[\mathbf{s}] \cdot\left\{\dot{\mathbf{u}_{i}}\right\}^{T}=0 .
$$

The backward difference form is used to discretize the time term, which can ensure the convergence of the difference iteration. The time step is $\Delta t$ :

$$
\left(\sum[\mathbf{k}]+\frac{\sum[\mathbf{s}]}{\Delta t}\right) \cdot\left\{\mathbf{u}_{i}\right\}^{T}=\sum[\mathbf{s}] \cdot\left\{u_{i-1}\right\}^{T} .
$$

Using the abovementioned finite-element form, the electric potential governing equation and the electroosmotic consolidation governing equation can be calculated. Here, Python is used to develop a special numerical calculation program for electroosmotic consolidation, which can calculate and solve the electric potential distribution and the pore water pressure.

\section{Example Analysis}

5.1. Two-Dimensional Effect of Electrode Unit. Consolidation of soil is a slow process. The aforementioned electroosmosis test only took 51 hours, and it is difficult to fully reflect the change of pore pressure during the electroosmosis process. 


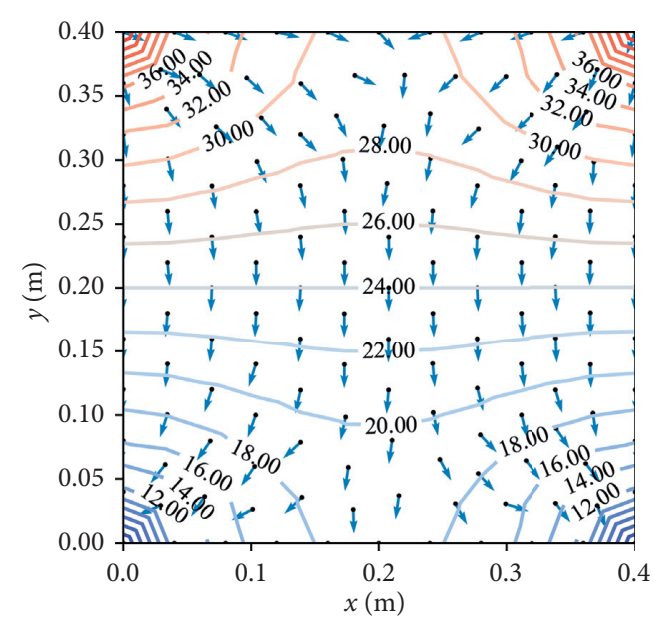

(a)

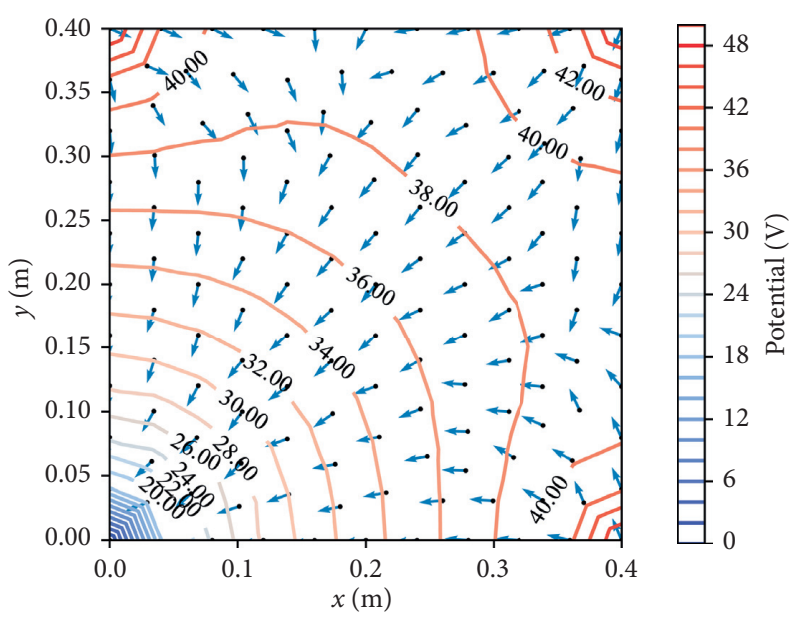

(b)

Figure 10: Gradient vector illustration of electric potential field. (a) Potential distribution of the symmetrical unit. (b) Potential distribution of the asymmetrical unit.

Therefore, a calculation example is carried out to calculate and analyse the electric potential field and electroosmotic consolidation of the electrode unit. The two-dimensional electroosmosis process must first determine the potential distribution of the unit, and the PyEcFem library is used to calculate this problem. The unit $L=0.4 \mathrm{~m}, W=0.4 \mathrm{~m}$, the applied voltage is $48 \mathrm{~V}$, and the potential distribution of the symmetric unit and the asymmetric unit is obtained, as shown in Figure 10. In the symmetric unit, the numbers of anodes and cathodes are equal, so the potential distribution is symmetrical (see Figure 10(a)). In the case of $W / L=1$, the potential distribution is significantly different from the uniform electric field, and the potential in the middle of the unit is lower than the potential at the electrode. At this time, if it is still calculated according to the one-dimensional electroosmotic consolidation theory, it will lead to an overestimation of the potential gradient. In the asymmetric unit (see Figure 10(b)), due to the asymmetry of the electrode properties, the potential distribution in this case cannot be simplified to a linear distribution, and the two-dimensional effect of the potential distribution is significant.

In actual engineering, when the distance between the electrodes of the same nature is much smaller than the distance between the electrodes of the opposite, in the case of symmetry, the distribution of the electric potential field is similar to the uniform electric field. In this case, the onedimensional electroosmotic consolidation model can be used to solve the problem. However, there is a lack of quantitative research. In order to study this problem, the calculations with different spacing ratios $(W / L=1,2,3,4,5)$ and $W$ remaining unchanged are carried out. The potential distribution is shown in Figure 11. It can be found that as the spacing ratio increases, the potentials' value distribution is getting closer and closer to the uniform electric field. When the electrode pitch ratio is 1 , the electric potential has a significant attenuation phenomenon. When the pitch ratio is equal to 5 , this phenomenon is basically eliminated.
5.2. Distribution Characteristics of Excess Pore Water Pressure. On the basis of obtaining the electric potential distribution, the calculation of electroosmosis consolidation can be determined. The soil electroosmosis coefficients $k_{e}=5 \times 10^{-9} \mathrm{~m}^{2} \cdot(s \cdot V)^{-1}, \quad k_{h}=2.5 \times 10^{-8} \mathrm{~ms}^{-1}, \quad$ and $C_{h}=5 \times 10^{-7} \mathrm{~m}^{2} \mathrm{~s}^{-1}$; the initial pore water pressure is $u(x, y, 0)=1.5 \mathrm{kPa}$, the total time is $10^{8}$ seconds, and the number of time steps is 10000 , so $\Delta t=10^{4}$ seconds. Figure 12 shows the pore water pressure distribution of the unit after electroosmosis is completed. There is an obvious difference between the pore pressure distribution of the symmetric unit and the asymmetric unit after electroosmosis. The negative pore pressure in electroosmosis is determined by the potential value. The more the anodes, the larger the negative pore pressure distribution area. The electroosmotic consolidation effect of asymmetric units is better than that of symmetric units.

The electroosmotic consolidation is established on the basis of Terzaghi's seepage consolidation theory. The consolidation speed of the soil is determined by the hydraulic permeability coefficient of the soil. The difference in electric potential distribution will not affect the consolidation speed, as shown in Figure 13(a). The average degree of consolidation curve of the symmetric unit completely coincides with the consolidation curve of the asymmetric unit. This conclusion is consistent with Esrig's theory [6]. The electric potential field mainly affects the pore pressure during the electroosmotic consolidation process. After the electroosmosis is completed, the average excess pore water pressure in the symmetric unit is $-23.962 \mathrm{kPa}$, while the average excess pore water pressure in the asymmetric unit is $36.068 \mathrm{kPa}$, as shown in Figure 13(b). The larger the number of anodes, the larger the average potential value of the unit and the stronger the drainage power. In the initial stage of electroosmosis, the symmetric unit contains two cathode drainage boundaries, and the outflow of pore water is higher than that of the asymmetric unit. In the middle and late stages of 


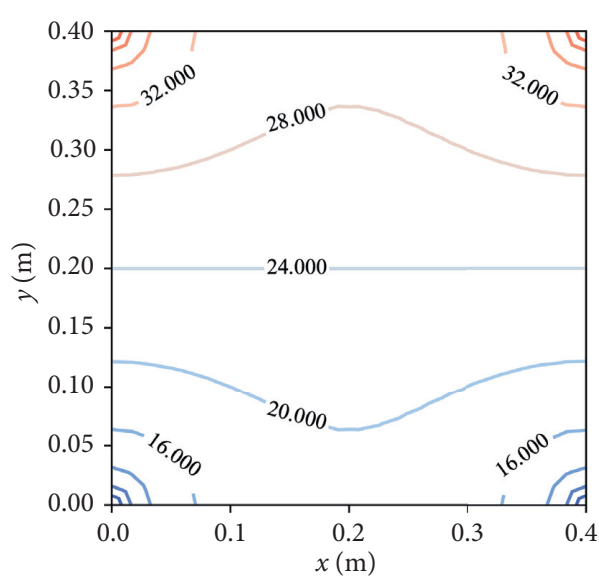

(a)

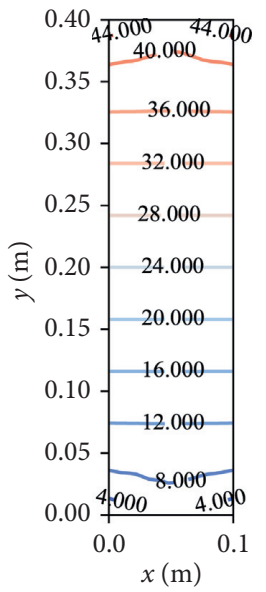

(d)

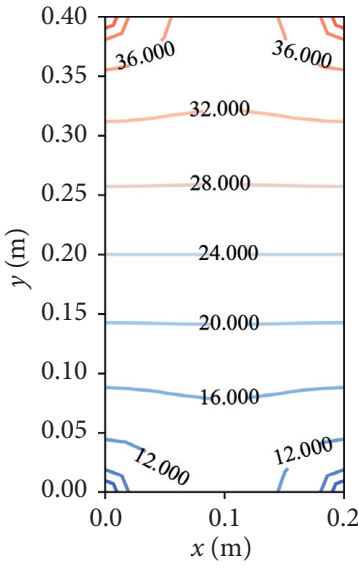

(b)

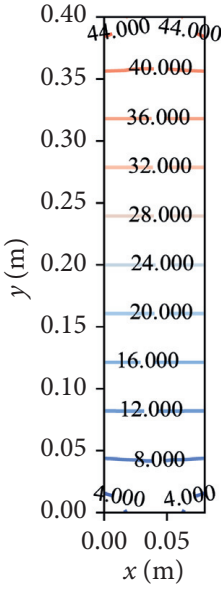

(e)

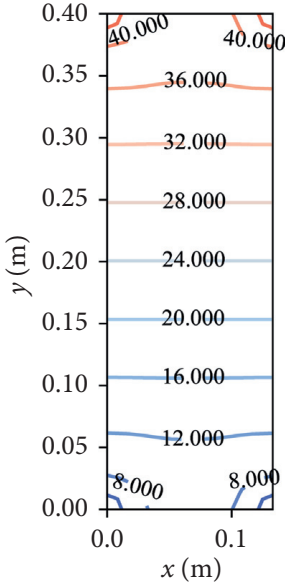

(c)

Figure 11: Potential contour plot of symmetric unit. (a) $W=0.4 \mathrm{ml}=0.4 \mathrm{~m}$. (b) $W=0.4 \mathrm{ml}=0.2 \mathrm{~m}$. (c) $W=0.4 \mathrm{~m}, L=0.133 \mathrm{~m}$. (d) $W=0.4 \mathrm{ml}=0.1 \mathrm{~m}$. (e) $W=0.4 \mathrm{ml}=0.08 \mathrm{~m}$.

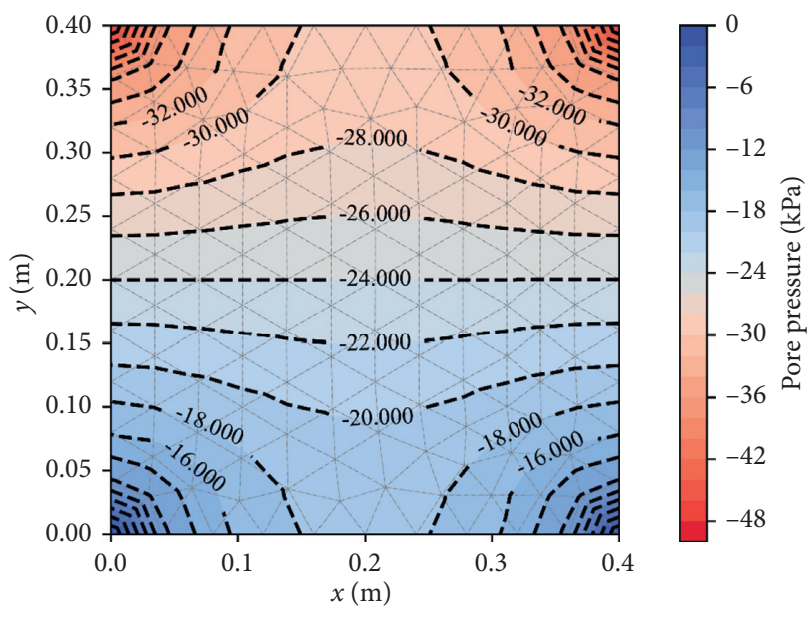

(a)

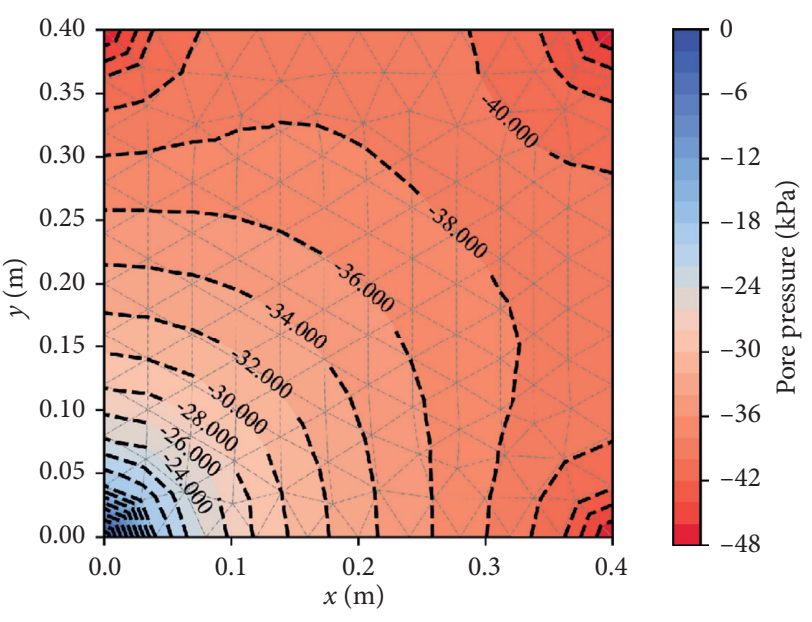

(b)

FiguRE 12: Colormap of excess pore water pressure distribution after electroosmosis. (a) The pore water pressure of the symmetric unit at time $10^{8} \mathrm{sec}$. (b) The pore water pressure of the asymmetric unit at time $10^{8} \mathrm{sec}$. 


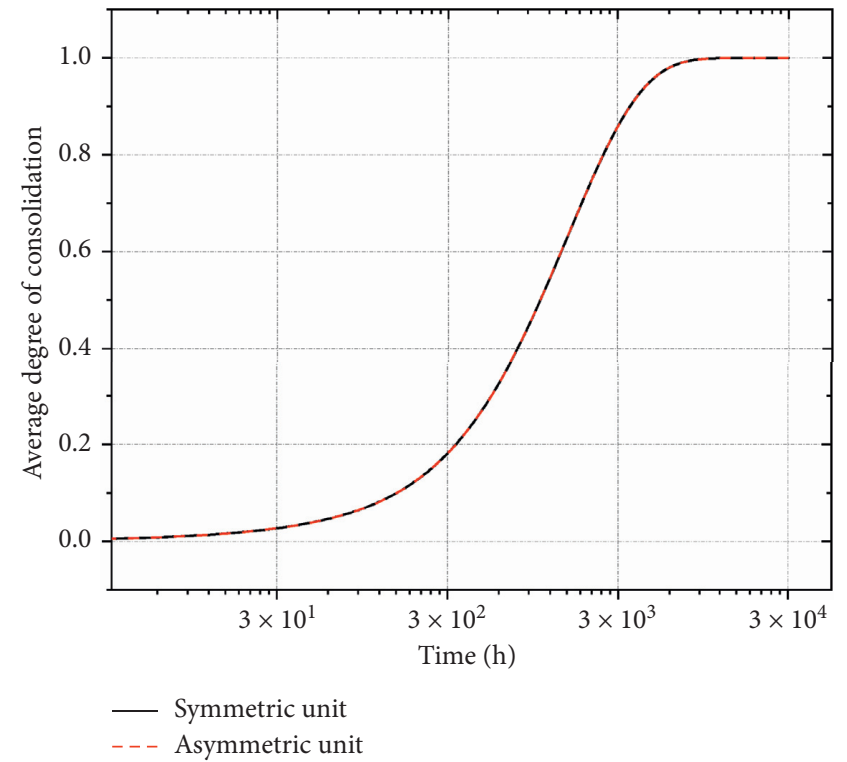

(a)

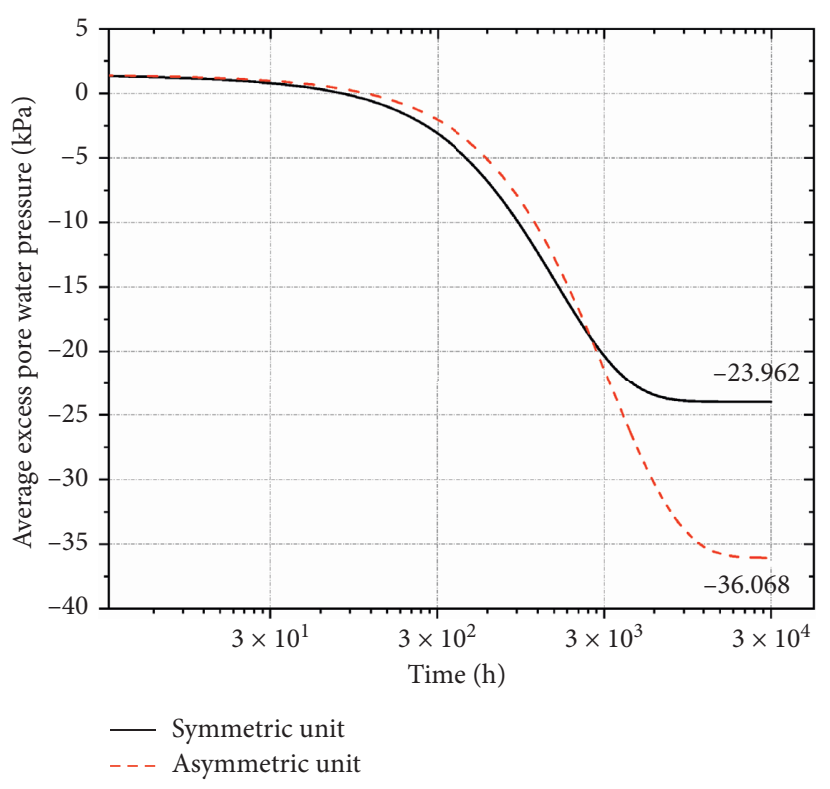

(b)

Figure 13: The average degree of consolidation curve and the average excess pore water pressure curve of the unit. (a) The unit average consolidation curve. (b) The curve of excess pore water pressure of unit.

electroosmosis, most of the pore water in the soil has been discharged, the influence of the number of drainage boundaries is reduced, and the driving force of the electric potential gradient becomes the main influence. The pore water pressure change rate of the symmetric unit gradually decreases, while the pore water pressure change rate of the asymmetric unit remains at a relatively high rate. The average excess pore water pressure of the asymmetric unit is $33.56 \%$ higher than that of the symmetric unit.

\section{Conclusions}

This paper focuses on the study of the two-dimensional electroosmotic consolidation characteristics when the electrode spacing is large, and the research object of the punctiform electrode unit is proposed. Electroosmotic consolidation test of the punctiform electrode unit is carried out; in the experiment, the electroosmotic consolidation characteristics of symmetric and asymmetric units and the difference in electric potential field are analyzed. Based on the continuity of water flow and the principle of linear superposition, the governing equation of the two-dimensional electroosmotic consolidation theory is established and the corresponding boundary conditions and initial conditions are given. The Galerkin method is used to discretize the governing equation, and the corresponding PDE numerical calculation module PyEcFem was developed by Python language; at the same time, the numerical solution of the example is carried out. The main conclusions are as follows:

(1) According to the difference of electrode polarity, the electrode unit can be divided into symmetrical unit and asymmetrical unit. The electroosmosis test shows that the drainage of the asymmetric model is higher than that of the symmetric model, but the symmetric model has a faster drainage rate in the initial stage. After the electroosmosis test is over, the water content of the asymmetric unit soil is lower than that of the symmetric unit, and more anodes in the asymmetric unit can promote soil reinforcement.

(2) The potential distribution of the electrode unit has obvious two-dimensional characteristics. In a symmetric unit, as the distance between the same electrodes decreases, the potential distribution tends to be uniform. When the spacing ratio is 5 , the potential field is basically close to a uniform electric field.

(3) With the same electroosmotic parameters and power-on time conditions, the electroosmotic reinforcement effect of asymmetric units is better than that of symmetric units. The negative pore pressure distribution in the asymmetric unit is relatively uniform, and the pore water pressure amplitude of the part near the cathode in the symmetric unit is lower than that near the anode. The unevenness of the soil after electroosmosis reinforcement is obvious. The potential distribution will not affect the consolidation rate, but it will affect the extreme value of negative pore water pressure. There are two drainage boundaries in the symmetric unit. In the initial stage of electroosmosis, the change rate of pore pressure is faster than that of the asymmetric unit. In the middle and late stages of electroosmosis, the rate of change of the pore pressure of the symmetric unit decreases more than that of the asymmetric unit. The increase in the number of anodes in electroosmosis consolidation can improve the effect of electroosmosis consolidation. 


\section{Data Availability}

The data used to support the findings of this study is available from the corresponding author upon request.

\section{Conflicts of Interest}

The authors declare that they have no conflicts of interest regarding the publication of this paper.

\section{Acknowledgments}

This study was supported by the Natural Science Foundation of China (no. 51778275) and Longyuan Young Innovative and Entrepreneurial Talents (team project no. 2020RCXM120).

\section{References}

[1] S. Micic, J. Q. Shang, K. Y. Lo, Y. N. Lee, and S. W. Lee, "Electrokinetic strengthening of a marine sediment using intermittent current," Canadian Geotechnical Journal, vol. 38, no. 2, pp. 287-302, 2001.

[2] I. L. Casagrande, "Electro-Osmosis in soils," Géotechnique, vol. 1, no. 3, pp. 159-177, 1949.

[3] J. Q. Shang, "Electrokinetic sedimentation: a theoretical and experimental study," Canadian Geotechnical Journal, vol. 34, no. 2, pp. 305-314, 1997.

[4] K. Y. Lo, I. I. Inculet, and K. S. Ho, "Electroosmotic strengthing of soft sensitive clays," Canadian Geotechnical Journal, vol. 28, no. 1, pp. 62-73, 2011.

[5] L. Bjerrum, J. Moum, and O. Eide, "Application of electroosmosis to a foundation problem in a Norwegian quick clay," Géotechnique, vol. 17, no. 3, pp. 214-235, 1967.

[6] M. I. Esrig, "Pore pressures, consolidation, and electrokinetics," Journal of the Soil Mechanics and Foundations Division, vol. 94, no. 4, pp. 899-921, 1968.

[7] R. W. Lewis and C. Humpheson, "Numerical analysis of electro-osmotic flow in soils," Journal of the Soil Mechanics \& Foundations Division, vol. 99, no. 3, pp. 603-616, 1973.

[8] T.-Y. Wan and J. K. Mitchell, "Electro-osmotic consolidation of soils," Journal of the Geotechnical Engineering Division, vol. 102, no. 5, pp. 473-491, 1976.

[9] J. Q. Shang, "Electroosmosis-enhanced preloading consolidation via vertical drains," Canadian Geotechnical Journal, vol. 35, no. 3, pp. 491-499, 1998.

[10] J. Q. Su and Z. Wang, "Theory of two-dimensional electroosmotic consolidation of soils," Rock and Soil Mechanics, vol. 25, no. 1, pp. 125-131, 2004.

[11] Y. Li, X. N. Gong, M. M. Lu et al., "Coupling consolidation theory under combined action of load and electro-osmosis," Chinese Journal of Geotechnical Journal, vol. 35, no. 2, pp. 388-394, 2013.

[12] $\mathrm{H}$. Wu, W. Wu, and L. Hu, "Numerical model of electroosmotic consolidation in clay," Géotechnique, vol. 62, no. 6, pp. 537-541, 2012.

[13] Y. D. Zhou, A. Deng, and J. Y. Fu, "Modelling electroosmosis-surcharge preloading combined consolidation of unsaturated soils," Computers and Geotechnics, vol. 114, Article ID 103145, 2019.

[14] A. Deng and Y. D. Zhou, "Modeling electroosmosis and surcharge preloading consolidation. II: validation and simulation results," Journal of Geotechnical and
Geoenvironmental Engineering, vol. 142, no. 4, Article ID 04015094, 2016.

[15] Y. Zhou, A. Deng, and C. Wang, "Finite-difference model for one-dimensional electro-osmotic consolidation," Computers and Geotechnics, vol. 54, no. 10, pp. 152-165, 2013.

[16] Y. D. Zhou, A. Deng, and Q. Lu, "A one-dimensional consolidation model considering large strain for unsaturated soil," Rock and Soil Mechanics, vol. 39, no. 5, pp. 1675-1681, 2018.

[17] Y. D. Zhou, J. Y. Fu, A. Deng et al., "A nonlinear model for electroomosis-vacuum preloading coupled consolidation," Chinese Journal of Rock Mechanics and Engineering, vol. 38, no. 8, pp. 1677-1685, 2019.

[18] L. J. Wang, Y. M. Wang, S. H. Liu et al., "Analytical solution for one-dimensional vertical electro-osmotic drainage under unsaturated conditions," Computers and Geotechnics, vol. 105, no. 9, pp. 27-36, 2018.

[19] L. J. Wang, P. H. Huang, S. H. Liu et al., “Analytical solution for nonlinear consolidation of combined electroosmosisvacuum-surcharge preloading," Computers and Geotechnics, vol. 121, no. 2, Article ID 103484, 2020.

[20] Y. Shen, J. T. Feng, Y. H. Ma et al., "Two-dimensional electroosmotic consolidation theory of nonlinear soil voltage distribution characteristics," Advances in Civil Engineering, vol. 2019, Article ID 7354873, 10 pages, 2019.

[21] X. Y. Yang and J. H. Dong, "A polymorphic inheritance calculation method of one-dimensional electro-osmotic consolidation considering effective potential attenuation," Chinese Journal of Rock Mechanics and Engineering, vol. 39, no. 12, pp. 2530-2539, 2020.

[22] Y. Li and X. N. Gong, "Experimental study on effect of soil salinity on electro-osmotic dewatering in soft clay," Chinese Journal of Geotechnical Engineering, vol. 33, no. 8, pp. 1254-1259, 2011. 